\title{
Spectroscopy and Photophysics of Monoazaphenanthrenes II. Ab initio Analysis of Molecular Parameters and Electronic Spectra
}

\section{DePerasińska AND J. Prochorow}

Institute of Physics, Polish Academy of Sciences

al. Lotników 32/46, 02-668 Warsaw, Poland

(Received September 11, 2003)

\begin{abstract}
The ground state structures of phenanthrene and its monoaza-derivatives, phenanthridine and 7,8-benzoquinoline were optimized using the $a b$ initio methods. Both methods of optimization are leading to the results, which are in good agreement with available experimental data. Calculated ground-state electric dipole moments in phenanthridine and 7,8-benzoquinoline were found to have antiparallel orientations, due to the different electronic charge distributions in these molecules. The energies of vertical electronic transitions from the ground to excited singlet states ( $S_{0} \rightarrow S_{n}$ transitions) and the corresponding oscillator strengths were calculated within the framework of configuration interaction-singles and time-dependent density functional theory. The last method was found to be more accurate in reproduction of experimental absorption spectra. Very interesting result of these computations is the change of relative orientation of the transition dipole moments for the two lowest $\pi \pi^{*}$ electronic transition in monoazaphenanthrenes from perpendicular in phenanthrene molecule to nearly parallel orientation in both monoazaphenanthrenes. The observed changes of molecular parameters and spectra can be related to the inductive effects of the substitution of nitrogen atom into the aromatic skeleton of phenanthrene.
\end{abstract}

PACS numbers: 82.20 .Wt, 31.15.Ar

\section{Introduction}

In physical organic chemistry the effect of substitution of the nitrogen atom into the aromatic ring system of hydrocarbon is known as the inductive effect of substituent [1]. In general, this effect is connected with the polarization of chemical bonds, due to shifts of their electron pairs in the direction of the electronegative group [2]. Nitrogen atom, which is more electronegative than the carbon atom, would attract electrons from the rest of the molecule, thus causing the inductive 
effect. The analysis of aromatic character of aza-derivatives of aromatic hydrocarbons has shown that the presence of nitrogen atom in the aromatic ring system increases its aromaticity [3].

The theoretical considerations of inductive effect, based on the first-order perturbation theory, predict rather small changes of the energy levels of hetero-conjugated molecule. In accordance with these considerations, experimentally determined electronic transition energies (corresponding to the two lowest $\pi \rightarrow \pi^{*}$ electronic transitions - to $L_{a}$ and $L_{b}$ states, in Platt's classification) in aza-hydrocarbons are only slightly changed in comparison to the transition energies observed for the parent hydrocarbon [1]. However, such a substitution (of nitrogen atom) brings about another new feature - the enhancement of the $L_{b}$ absorption band. The low intensity of this band in the aromatic hydrocarbon is attributed to the pairing property of the hydrocarbon molecular orbitals. On N-substitution this property is lost.

From the photophysical point of view, it is also important that after substitution of nitrogen atom into the aromatic hydrocarbon the lone-pair electrons ( $n$ electrons of $\mathrm{N}$ atom) are replacing two $\mathrm{C}-\mathrm{H}$ bonding electrons of hydrocarbon. As a consequence, in aza-hydrocarbons, in addition to the $\pi \rightarrow \pi^{*}$ electronic transitions (present in parent hydrocarbons), electronic transitions of the new type are expected to appear. These new transitions called $n \rightarrow \pi^{*}$ transitions are symmetry allowed and polarized perpendicularly to the molecular plane. In aza-benzenes and aza-naphthalenes $n \rightarrow \pi^{*}$ transitions are observed as either separate bands at lower energy than the bands corresponding to $\pi \rightarrow \pi^{*}$ transitions, or as shoulders on $\pi \rightarrow \pi^{*}$ absorption band [1]. However, in the case of polycyclic aza-aromatics the absorption bands corresponding to the $n \rightarrow \pi^{*}$ transitions are hidden under the absorption bands corresponding to the $\pi \rightarrow \pi^{*}$ transitions $[1,4-7]$.

Polycyclic aromatic hydrocarbons together with corresponding polycyclic aza-aromatics form the class of molecules, which are of importance in astrophysics and life sciences [8-11], but the properties of polycyclic aza-aromatics are not yet well recognized and understood. In this work we present the results of ab initio calculations of the properties of two azaphenanthrenes, phenanthridine (PHN) and 7,8-benzoquinoline (BQ), and of their parent hydrocarbon - phenanthrene (PH), which serves as a reference molecule.

The experimental data for phenanthridine and 7,8-benzoquinoline are relatively wide [12] and include: X-ray structures [13], IR spectra [12], the absorption and fluorescence spectra of free bases and their conjugated acid forms of both these molecules in aqueous solutions [6], values of ionization potentials [14]. Dipole moments in the ground and excited states of 7,8-benzoquinoline were derived from the solvent effect on absorption and fluorescence spectra [7]. Recently the IR spectrum of the argon matrix isolated BQ was published [11] and the analysis of the solvent effects on electronic absorption and fluorescence spectra and on photophysical parameters of both azaphenanthrenes and phenanthrene were presented [15]. 
The comparison of the electronic absorption spectra of PHN and BQ with the spectrum of phenanthrene indicates that the energies of the $S_{0} \rightarrow S_{1}$ transitions are only slightly different while the extinction coefficient is much larger for azaphenanthrenes [15].

Up to now, however, the theoretical and computational works were concentrated almost exclusively on the phenanthrene molecule $[9,16,17-21]$ with one exception - recently the interpretation of IR spectrum of 7,8-benzoquinoline was performed by ab initio calculations [11]. Most of the advanced, ab initio computations for $\mathrm{PH}$ are connected with the interpretation of its vibrational spectrum [16, 17-19]. Still, however, the understanding of the electronic spectra of $\mathrm{PH}$ on the level of $a b$ initio calculations $[9,20,21]$ is not satisfactory.

In the present paper we are computing the optimized geometry of phenanthrene and both azaphenanthrenes and compare the different molecular properties corresponding to these structures in search of the effects caused by the nitrogen atom substitution into the aromatic ring system.

\section{Computational details}

The schematic structures of the phenanthrene, phenanthridine and 7,8-benzoquinoline, together with the bond numbering, are presented in Fig. 1.

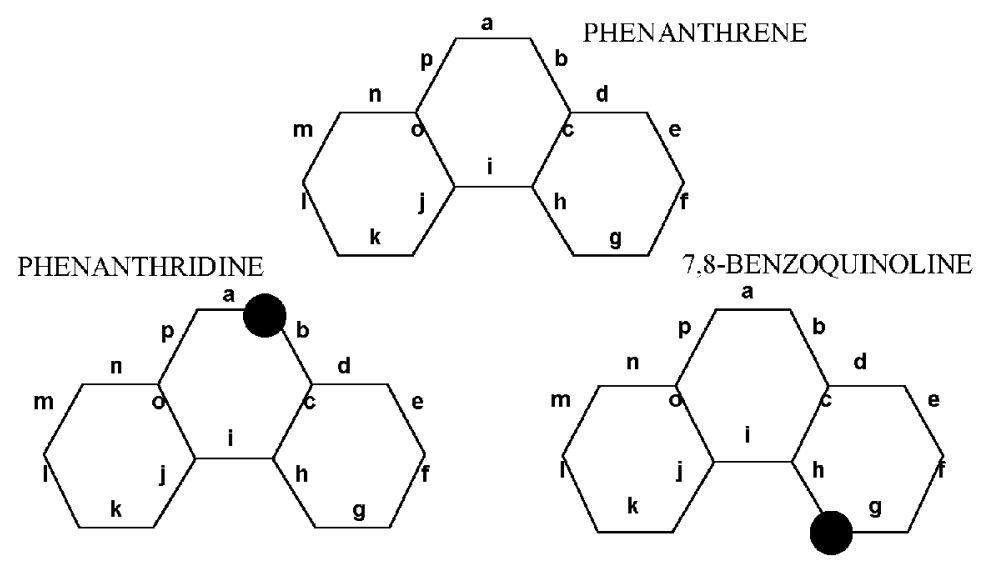

Fig. 1. Structures of investigated molecules and numbering of the bonds. Black circles indicate the position of nitrogen atom.

The geometry of each of these molecules were optimized by restricted Hartree-Fock at 6-31G(d) level (RHF/6-31G(d)) and density functional theory at B3LYP/6-31G(d) level (DFT B3LYP/6-31G(d)). The optimization calculations were followed by the calculations of the vibrational frequencies in order to verify, 
whether the obtained stationary points are the true minima. The energies of electronic transitions from the ground to the excited states and corresponding oscillator strengths (for the structures optimized in the ground state) were determined by restricted configuration interaction-singles at 6-31G(d) level (RCIS/6-31G(d)) and time-dependent density functional theory at B3LYP/6-31G(d) level (TD DFT B3LYP/6-31G(d)) methods.

All calculations have been carried out using the GAUSSIAN 98 programs $[22]$

\section{Results}

\subsection{Ground state optimized geometry}

All three molecules under consideration, i.e. $\mathrm{PH}, \mathrm{PHN}$, and $\mathrm{BQ}$, in their RHF/6-31G(d), and DFT/B3LYP/6-31G(d) optimized geometry have planar structures. Bond lengths obtained for the optimized geometry (within the framework of both these methods) are presented in Fig. 2 together with the experimental crystallographic data taken from [13]. It is seen that the differences between bond lengths determined by the both methods are not exceeding $0.02 \AA$ for all three molecules. However, a comparison of the results of calculations with experimental data shows that for $\mathrm{PH}$ and PHN molecules they also agree within the $0.02 \AA$ limit, but for BQ experimental and calculated bond lengths can differ by $0.045 \AA$ for $\mathbf{a}, \mathbf{b}, \mathbf{j}, \mathbf{k}$ and $\mathbf{m}$ bonds. At this point we must mention, however, that similar differences can be found for the bond lengths of phenanthrene molecule obtained in different experiments $[13,18,19,23]$.

A close inspection of Fig. 2 reveals that the largest changes of the bond lengths are observed for the bonds $\mathbf{a}$ and $\mathbf{b}$ in $\mathrm{PHN}$ and $\mathbf{h}$ and $\mathbf{g}$ in $\mathrm{BQ}$. If we refer to Fig. 1 then it turns out that those are bonds in which nitrogen $(\mathrm{N})$ atom is participating. Calculated decrease in the length of bond a of PHN in comparison to $\mathrm{PH}$ is $0.066 \AA$ (RHF calculations) and $0.060 \AA$ (DFT calculations). Bond b in PHN is getting shorter by either $0.060 \AA$ (RHF) or $0.051 \AA$ (DFT). In the case of $\mathrm{BQ}$ molecule the corresponding changes are $0.069 \AA$ (RHF) and $0.060 \AA$ (DFT) for $\mathbf{h}$ bond and $0.066 \AA$ (RHF) and $0.059 \AA$ (DFT) for $\mathbf{g}$ bond.

Therefore, the substitution of $\mathrm{N}$ atom into the phenanthrene aromatic system results in the relatively large decrease (by $\sim 0.06 \AA$ ) in the length of bonds formed with participation of $\mathrm{N}$ atom (instead of $\mathrm{C}$ atom). This quantity is slightly larger than the experimental results which estimate this decrease as $\sim 0.04 \AA$.

The changes of bond lengths are accompanied by the changes of the angles between the bonds and it leads to the deformations of the $\mathrm{PH}$ rings. In the case of PHN the largest changes $\left(c a . \pm 3^{\circ}\right)$ are those of the angles $(\mathbf{a}, \mathbf{b}),(\mathbf{a}, \mathbf{p}),(\mathbf{b}, \mathbf{d})$ and $(\mathbf{b}, \mathbf{c})$, while in the case of BQ these changes are slightly smaller $\left(c a . \pm 2.5^{\circ}\right)$, but more extended topologically - beside the angles $(\mathbf{g}, \mathbf{f}),(\mathbf{g}, \mathbf{h}),(\mathbf{h}, \mathbf{c})$ and $(\mathbf{h}, \mathbf{i})$ they include also $(\mathbf{c}, \mathbf{d})$ and $(\mathbf{b}, \mathbf{d})$ angles. In other words in the case of PHN the 

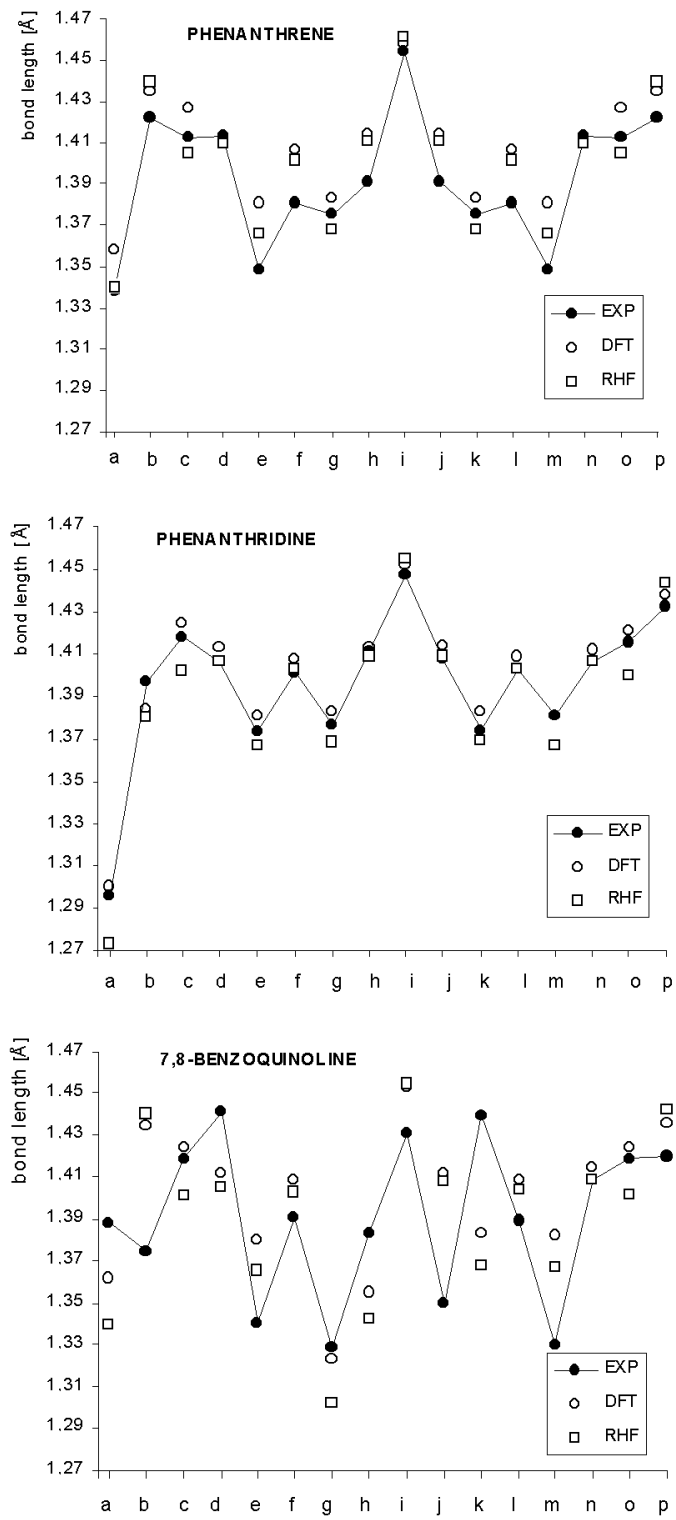

Fig. 2. Comparison of experimental values [13] of the bond lengths $(-\bullet-)$ with their values calculated with the use of DFT/B3LYP/6-31G(d) (o) and RHF/6-31G(d) ( $\square$ ) method, for phenanthrene, phenanthridine, and 7,8-benzoquinoline (numbering of the bonds as in Fig. 1).

$\mathrm{N}$-substitution causes the changes of $\mathrm{CNC}$ and $\mathrm{CCN}$ angles while in the case of $\mathrm{BQ}$ also the two CCC angles undergo such changes. In PHN, the N-substitution in the central ring of phenanthrene deforms this ring and changes the angle between 
the central ring and neighboring external ring. In BQ, the N-substitution in the external ring of phenanthrene deforms this ring, changes the angle between the external and central ring and changes also the CCC angle in the central ring.

Therefore the substitution of $\mathrm{N}$ atom to the phenanthrene rings leads to the non-negligible geometric changes in both azaphenanthrenes and results in the reduction of $C_{2 v}$ symmetry characterizing the parent molecule of phenanthrene.

\section{3. . Charge distributions and dipole moments in the ground state}

The reduction of symmetry of phenanthrene in azaphenanthrenes is especially clearly seen when the charge distributions and electric dipole moments of these molecules are compared. The substitution of $\mathrm{N}$ atom into the phenanthrene aromatic system changes the charge distribution in the manner shown in Fig. 3 a and $\mathrm{b}$ for PHN and BQ molecules, respectively. It is seen that these changes are large but simultaneously are of local character, limited to the part of molecule. The presence of $\mathrm{N}$ atom in the place of $\mathrm{C}$ atom in phenanthrene system is equivalent to the increase in negative charge in the place of substitution (in Fig. 3 atom 14 in PHN and atom 9 in BQ, respectively). This change is accompanied by the increase in positive charge on neighboring $\mathrm{C}$ atoms (atoms 1 and 13 for PHN and 8 and 10 for $\mathrm{BQ}$, respectively).

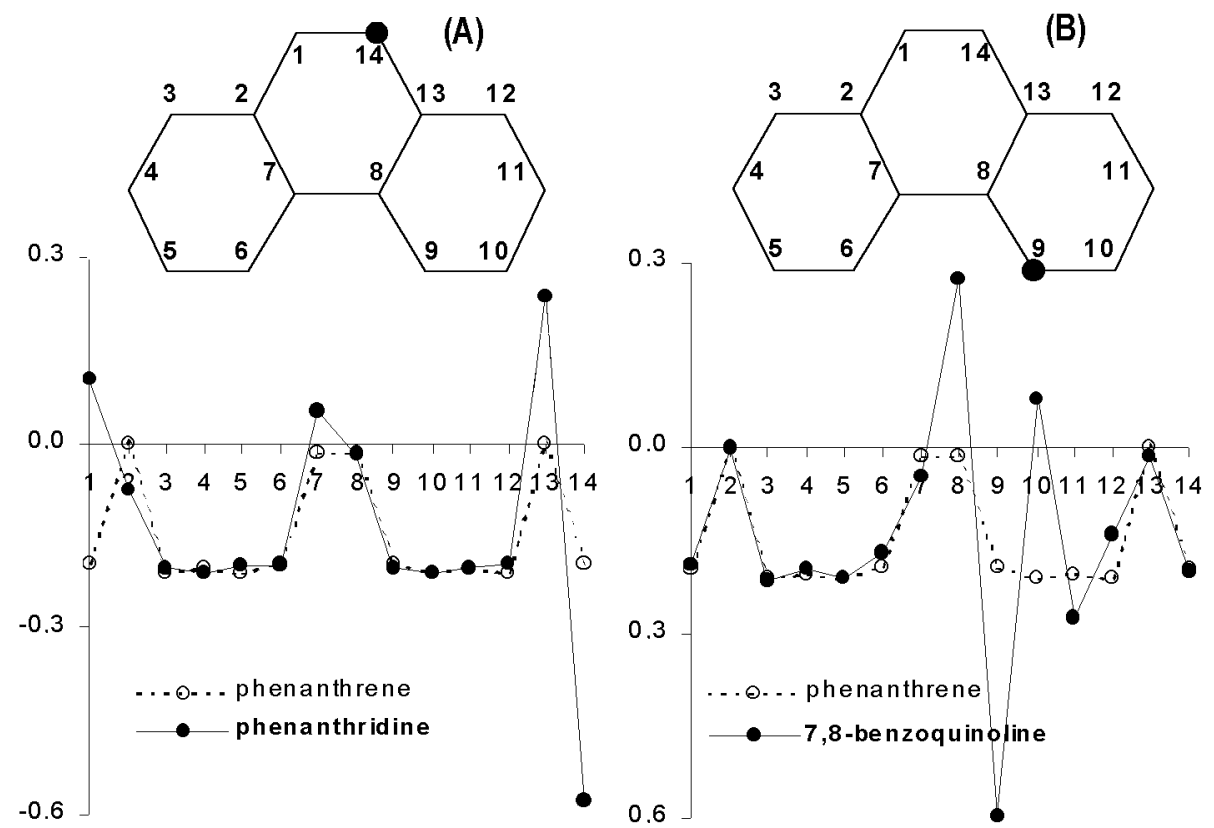

Fig. 3. Comparison of the results of RHF/6-31G(d) calculations of the charge distribution in phenanthridine $(A)$ and in 7,8-benzoquinoline $(B)$ molecules with the charge distribution in phenanthrene molecule. 


\begin{tabular}{|l|c|c|}
\hline PHENANTHRENE & PHENANTRIDINE & 7,8-BENZOQUINOLINE \\
RHF: $\mu=0.02 \mathrm{D}$ & RHF: $\mu=2.44 \mathrm{D}$ & RHF: $\mu=1.84 \mathrm{D}$ \\
DFT: $\mu=0.04 \mathrm{D}$ & DFT: $\mu=2.22 \mathrm{D}$ & EXP: $\mu=0.52 \mathrm{D}[7]$
\end{tabular}

Fig. 4. Calculated values and directions $(-\rightarrow+)$ of the electric dipole moment in the ground state of $\mathrm{PH}, \mathrm{PHN}$, and $\mathrm{BQ}$ molecules.

Charge distribution in both investigated azaphenanthrenes becomes asymmetric. The asymmetry leads to the appearance of the electric dipole moments whose values are larger than the very small dipole moment of $\mathrm{PH}$ molecule (see Fig. 4). These dipole moments are directed along the line, which forms an angle of $c a .30^{\circ}$ with the direction of the dipole moment of $\mathrm{PH}$. It is also seen from Fig. 4 that the values of the dipole moment calculated by RHF and DFT methods are similar and that the calculated dipole moment for 7,8-benzoquinoline is much larger as compared to the experimentally estimated value [7], which probably should be subjected to verification.

\subsection{Molecular orbitals}

In Fig. 5 the RHF orbital energy diagrams of the highest occupied molecular orbitals (HOMO) and the lowest unoccupied molecular orbitals (LUMO) for all three molecules under consideration are given. The shapes of the frontier orbitals are also visualized. As it can be seen, the energies of all levels in azaphenanthrenes are systematically lower than the energies of the levels in phenanthrene. In terms of the first-order perturbation theory such behavior is a symptom of the inductive effect of substituent for alternate hydrocarbons [1].

The energies of HOMO orbitals: $7.67 \mathrm{eV}$ for PH, $8.10 \mathrm{eV}$ for PHN, and $7.81 \mathrm{eV}$ for $\mathrm{BQ}$ can be compared to the ionization potentials of these molecules, which were experimentally determined as 7.89 [12, 23], 8.31 and $8.04 \mathrm{eV}[12,14]$, respectively. And this regular shift of $c a .0 .2 \mathrm{eV}$ can be considered as a quite good agreement between calculations and experiment.

However such agreement is absent for the electron affinity - the calculated energy of LUMO orbital of phenanthrene, $2.72 \mathrm{eV}$, is much larger than the experimental value of electron affinity of this molecule estimated as $<0.3 \mathrm{eV}[12,24]$. Such discrepancy holds also in the case of the energy gap between HOMO and 


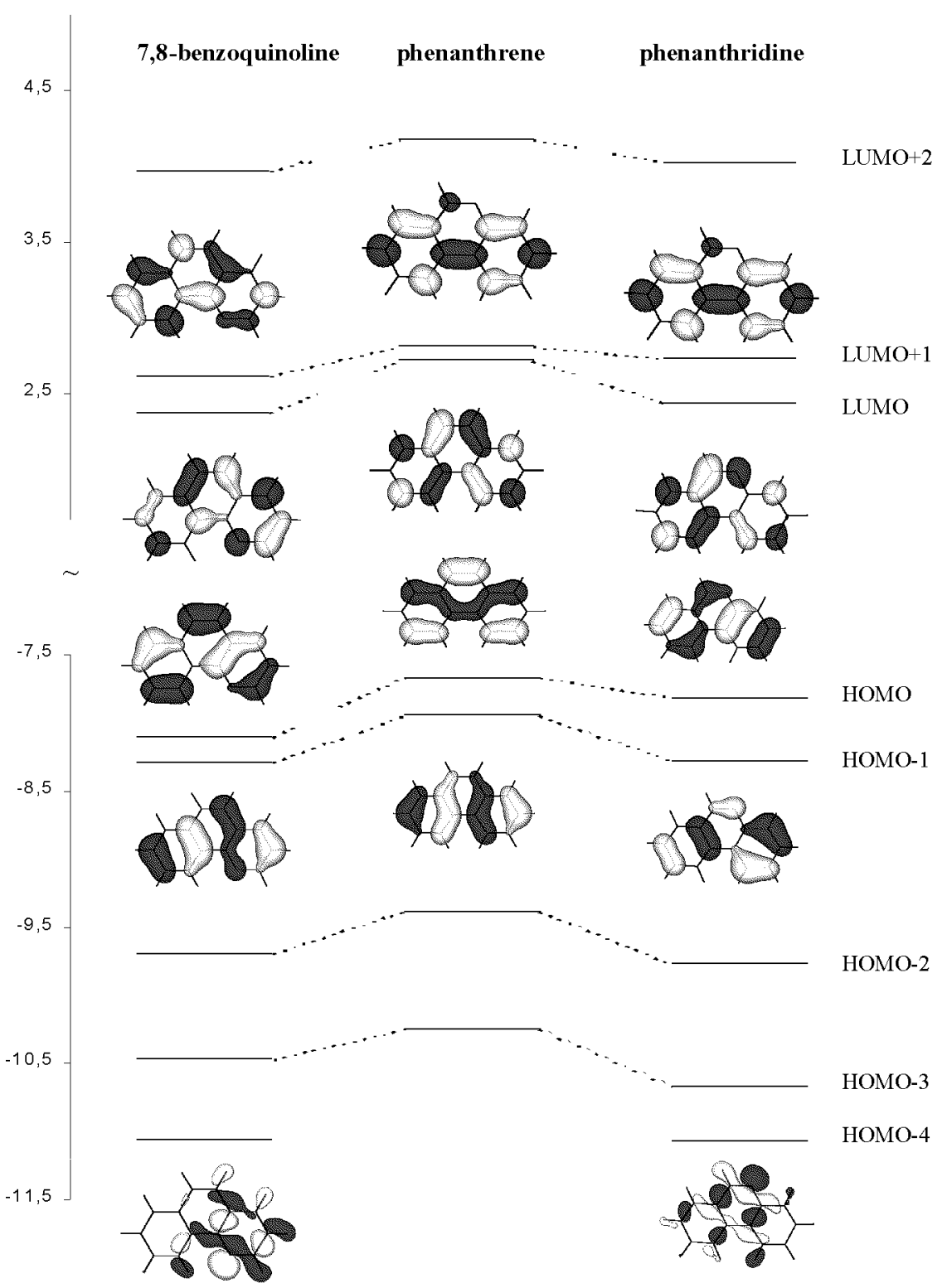

Fig. 5. Orbital energy diagram for phenanthrene and azaphenanthrenes (energy scale in $\mathrm{eV}$ ) and the cross-sections of frontier orbitals (the black and grey regions reflect the nodal characteristic of the orbital symmetry).

LUMO, which is too large. This is known as a defect of ab initio RHF calculation $[9,25,26]$ which leads to the overestimation of the energies of electronic transitions. 
It can also be noticed (cf. the orbital shapes) that there is a rather close correspondence between the LUMO (as well as LUMO+1) of PH molecule and LUMOs (and respectively LUMOs+1) of BQ and PHN molecules. On the other hand, the HOMO and HOMO- 1 of the both, $\mathrm{BQ}$ and $\mathrm{PHN}$, are clearly the combinations of HOMO and HOMO-1 of PH molecule. And finally, we notice that in $\mathrm{PH}$ molecule there is no equivalent of the $\mathrm{HOMO}-4$ observed in either $\mathrm{BQ}$ or $\mathrm{PHN}$ molecules. The shape of HOMO-4 in both molecules indicates that this orbital can be treated as the "classical" $n$-orbital, although it is partially delocalized over the other atoms of the system.

We should mention that the orbital diagram obtained within the framework of DFT method is qualitatively similar to that discussed above, with very similar shapes of orbitals. The most important difference between both methods is that RHF-calculated HOMO-4 orbital becomes HOMO-2 within DFT method.

\subsection{Electronic spectra}

The HOMO orbital energies obtained within DFT method are by $\sim 2 \mathrm{eV}$ higher than the RHF-calculated energies. At the same time, however, the energy gap between HOMO and LUMO calculated by DFT method is lower than that calculated with the use of RHF method. As a result, the energies of electronic transitions calculated by means of DFT method are lower and much closer to the experimental observations than those obtained within RHF method. The following values are illustrating this fact: (a) experimentally observed wavenumbers of the $(0,0)$ band of the absorption of $\mathrm{PH}, \mathrm{BQ}$, and $\mathrm{PHN}$ in $n$-hexane solution are 28918,28935 , and $29137 \mathrm{~cm}^{-1}$, respectively [15], (b) energies of $S_{0} \rightarrow S_{1}$ transition calculated by RCIS method for PH, BQ, and PHN are $\Delta E=41456,41996$, and $42382 \mathrm{~cm}^{-1}$, respectively, and (c) transitions energies calculated by DFT method: are 32457,32107 , and $32703 \mathrm{~cm}^{-1}$ for $\mathrm{PH}, \mathrm{BQ}$, and PHN, respectively. It is evident that DFT results are much closer to the experiment, and similar relations between ab initio RCIS, TD DFT and experimental transition energies have been obtained for many other molecules [20, 21].

The energies of $S_{0} \rightarrow S_{n}$ transitions calculated by TD DFT method for PH, PHN, and BQ molecules are collected in the Table, while in Fig. 6 a comparison of calculated and experimental absorption spectra [15] is given. In the reconstruction of calculated spectra we used the following procedure. Each calculated electronic transition is described by definite values of energy of transition and oscillator strength (i.e. the length of the vertical stick, corresponding to the given transition energy is proportional to the oscillator strength of this transition). The vertical lines were then replaced by Gaussian contours. The maximum of contour is placed at the calculated transition energy, its height is equal to the calculated oscillator strengths. The value of $1000 \mathrm{~cm}^{-1}$ was taken as the width of each contour. The envelope of such Gaussian contours composes the calculated absorption spectrum, which can be now compared with broad and diffused experimental spectra. 
TABLE

Energies $(\Delta E)$ of electronic $S_{1} \rightarrow S_{n}$ transitions and their oscillator strengths $(f)$ calculated with the use of TD DFT B3LYP/6-31G(d) method. Abbreviations of $A_{n}, B_{n}$ and $A^{\prime}, A^{\prime \prime}$ refer to the group elements in $C_{2 v}$ and $C_{s}$ symmetry representations, respectively.

\begin{tabular}{c|c|c|c|c|c|c|c|c}
\hline \hline \multicolumn{3}{c|}{ phenanthrene } & \multicolumn{3}{|c|}{ phenanthridine } & \multicolumn{3}{|c}{7,8 -benzoquinoline } \\
\hline $\begin{array}{c}\text { sym- } \\
\text { metry }\end{array}$ & $\begin{array}{c}\Delta E \\
{\left[\mathrm{~cm}^{-1}\right]}\end{array}$ & $f$ & $\begin{array}{c}\text { sym- } \\
\text { metry }\end{array}$ & $\begin{array}{c}\Delta E \\
{\left[\mathrm{~cm}^{-1}\right]}\end{array}$ & $f$ & $\begin{array}{c}\text { sym- } \\
\text { metry }\end{array}$ & $\begin{array}{c}\Delta E \\
{\left[\mathrm{~cm}^{-1}\right]}\end{array}$ & $f$ \\
\hline$A_{1}$ & 32457 & 0.001 & $A^{\prime}$ & 32703 & 0.007 & $A^{\prime}$ & 32107 & 0.044 \\
$B_{2}$ & 34447 & 0.068 & $A^{\prime \prime}$ & 34513 & 0.001 & $A^{\prime}$ & 34338 & 0.030 \\
$A_{1}$ & 38936 & 0.125 & $A^{\prime}$ & 34555 & 0.059 & $A^{\prime \prime}$ & 35721 & 0.002 \\
$B_{2}$ & 41220 & 0.746 & $A^{\prime}$ & 39021 & 0.105 & $A^{\prime}$ & 38345 & 0.104 \\
$A_{1}$ & 42082 & 0.003 & $A^{\prime \prime}$ & 39519 & 0.000 & $A^{\prime \prime}$ & 38607 & 0.000 \\
$B_{2}$ & 42423 & 0.025 & $A^{\prime}$ & 40821 & 0.491 & $A^{\prime}$ & 40344 & 0.373 \\
$B_{2}$ & 47057 & 0.008 & $A^{\prime}$ & 42821 & 0.072 & $A^{\prime}$ & 43061 & 0.016
\end{tabular}

It is seen from Fig. 6 that the procedure applied to the TD DFT-computed electronic transitions reproduces quite well the measured absorption spectra of all three molecules under investigations. The 10 lowest calculated electronic transitions are leading to the spectrum (calculated one) composed of four bands with the intensity distribution similar to the experimentally observed.

Furthermore we notice that the present calculations are also reproducing experimentally observed enhancement of $S_{0} \rightarrow S_{1}$ transition in azaphenanthrenes, as compared to its intensity in parent phenanthrene molecule. Such effect is caused by the reduction of symmetry of phenanthrene after N-substitution, which results in the lost of the pairing property of parent hydrocarbon molecular orbitals. Each of two lowest $\pi \rightarrow \pi^{*}$ transitions in azaphenanthrenes is described by the configuration interactions of four electronic configuration (HOMO-1 $\rightarrow$ $\mathrm{LUMO}+1, \mathrm{HOMO}-1 \rightarrow \mathrm{LUMO}, \mathrm{HOMO} \rightarrow \mathrm{LUMO}+1$ and $\mathrm{HOMO} \rightarrow \mathrm{LUMO}$ ), while $L_{b}$ and $L_{a}$ excited states in parent $\mathrm{PH}$ molecule are described only by the pairs of configurations (HOMO-1 $\rightarrow$ LUMO, $\mathrm{HOMO} \rightarrow \mathrm{LUMO}+1$ ) and (HOMO $\rightarrow$ LUMO, $\mathrm{HOMO}-1 \rightarrow \mathrm{LUMO}+1)$, respectively.

These differences in configuration interactions in azaphenanthrenes and in phenanthrene are reflected also by the changes of the relative polarization of two low-lying $\pi \rightarrow \pi^{*}$ transitions. The angle between directions of transition dipole moments for these two electronic transitions states is $90^{\circ}$ for phenanthrene, while in the case of azaphenanthrenes it is close to $0^{\circ}$. It comes out from our calculations that, in the case of PHN, transition dipole moments for electronic transitions under consideration form an angle of $c a .30^{\circ}$ with the long axis of molecule (which is parallel to the molecular bond $\mathbf{i}$ (cf. Fig. 1)). In the case of BQ this angle is predicted to be near $0^{\circ}$. 


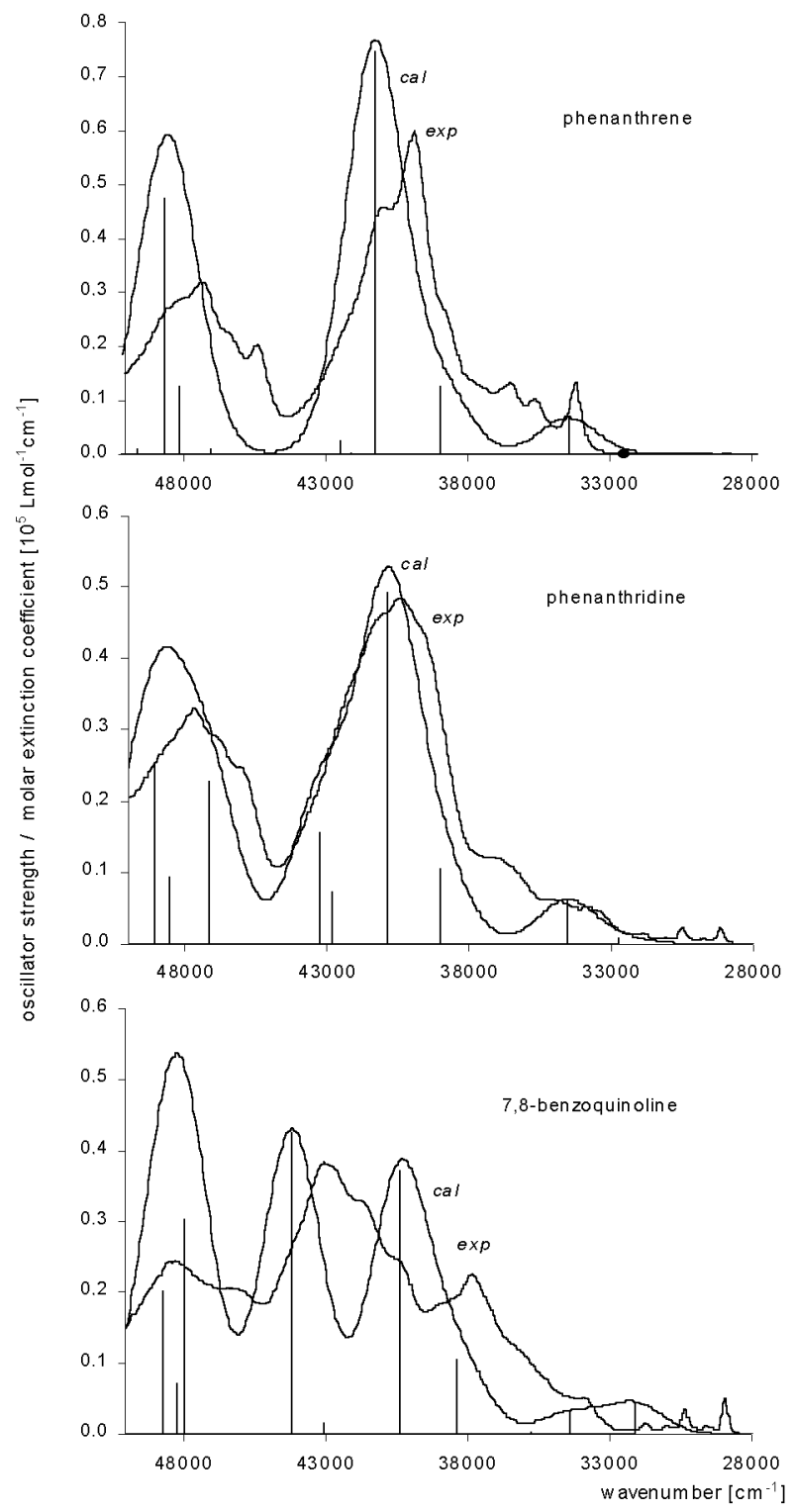

Fig. 6. Electronic absorption spectra of $\mathrm{PH}, \mathrm{PHN}$, and BQ molecules. Experimentally observed spectra (exp) in $n$-hexane solution [15] are compared with the results of calculations (cal). The vertical lines correspond to the TD DFT-calculated energies of $S_{0} \rightarrow S_{n}$ electronic transitions (see the text for details).

Another result of N-substitution is the appearance of $n \rightarrow \pi^{*}$ transitions (see the Table) with a small oscillator strength and transition dipole moment perpendicular to the molecular plane $\left(A^{\prime \prime}\right)$. Such transition is identified as $S_{0} \rightarrow S_{2}$ 
transition in the case of PHN and as $S_{0} \rightarrow S_{3}$ transition in the case of BQ. Up to now, these electronic transitions have not been experimentally identified [15].

\section{Concluding remarks}

The results of analysis and calculations performed in this work demonstrate that the substitution of the nitrogen atom in the phenanthrene aromatic ring system has a character of the inductive effect. Such substitution changes the electronic and geometrical structure of molecular system of azaphenanthrenes (in comparison to the phenanthrene) and leads to the appearance of the electric dipole moments whose values are larger than the very small dipole moment of phenanthrene. The results of calculations are reproducing quite well the measured absorption spectra and confirm the conclusions of the experimental work [15] that the first excited singlet state of azaphenanthrenes is of $\pi \pi^{*}$-type (at the same time the "phenanthrenes' $L_{b}$ character" of this state is lost).

Very interesting and experimentally very challenging is the result (delivered by the both ab initio, RCIS and TD DFT, calculations) which indicates a near-parallel orientation of the transitions dipole moments of electronic transitions to the two lowest $\pi \pi^{*}$ states of azaphenanthrenes. Another result, which can be of importance for understanding the photophysics of azaphenanthrenes in solutions, is the prediction that the lowest $n \pi^{*}$ state is the second, $S_{2}$, and the third, $S_{3}$, excited state within the singlets manifold of $\mathrm{PHN}$ and $\mathrm{BQ}$, respectively. Such ordering might be important, especially in view of the conclusion (inferred from the observations of fluorescence and its decays [15]) according to which a strongly polar and hydrogen-bonding solvent (such as methanol) induces in these two molecules very different quantitative changes of the rate constants of nonradiative transitions in the first excited state. The last point clearly calls for the advanced calculations of the optimized structures and parameters of excited states of azaphenanthrenes, which is the next step in continuation of the present work.

\section{Acknowledgment}

We gratefully acknowledge partial financial support of this work by the State Committee for Scientific Research under project 5 P03B 13620.

\section{References}

[1] M. Klessinger, J. Michl, Excited States and Photochemistry of Organic Molecules, VCH Publishers, Inc. New York 1995, pp. 18, 73, 93.

[2] V.I. Minkin, Pure Appl. Chem. 71, 1919 (1999).

[3] M. Cyrański, T.M. Kygowski, Tetrahedron 52, 13795 (1996).

[4] J. Prochorow, I. Deperasiska, O. Morawski, Chem. Phys. Lett. 316, 24 (2000). 
[5] J. Prochorow, I. Deperasińska, O. Morawski, J. Mol. Struct. 555, 97 (2000).

[6] C.J. Marzzacco, G. Deckey, R. Colarulli, G. Siuzdak, A.M. Halpern, J. Phys. Chem. 93, 2935 (1989).

[7] I. Janic, A. Kawski, Bull. Acad. Pol. Sci., Ser. sci. mat. astr. phys. XX, 235, 519 (1972).

[8] R. Dabestani, I.N. Ivanov, Photochem. Photobiol. 70, 10 (1999).

[9] L.D. Betowski, M. Enlow, L. Riddick, Comput. Chem. 26, 371 (2002).

[10] F. Salama, C. Joblin, L.J. Allamandola, J. Chem. Phys. 101, 10252 (1994).

[11] A.L Mattioda, D.M. Hudgins, C.W. Bauschlicher, Jr., M. Rosi, L.J. Allamandola, J. Phys. Chem. A 107, 1486 (2003).

[12] http://webbook.nist.gov/chemistry/form-ser.html.

[13] R. Kiralj, B. Kojic-Prodic, M. Zinic, S. Alhodzic, N. Trinajstyc, Acta Crystallogr. $B$ 52, 823 (1996).

[14] N.S. Hush, A.S. Cheung, P.R. Hilton, J. Electron Spectrosc. Relat. Phenom. 7, 385 (1975).

[15] M. Norek, J. Dresner, J. Prochorow, Acta Phys. Pol. A 104, 425 (2003).

[16] E. Cane, A. Miani, P. Palmieri, R. Tarroni, A. Trombetti, Spectrochim. Acta A 53, 1839 (1997).

[17] S.R. Langhoff, J. Phys. Chem. 100, 2819 (1996).

[18] J.M.L. Martin, J. El-Yazi, J.-P. Francois, J. Phys. Chem. 100, 15358 (1996).

[19] I. Bandyopadhyay, S. Manogaran, J. Mol. Struct. (Theochem) 496, 107 (2000).

[20] K. Nishimoto, Internet Electronic Journal of Molecular Design 1, 572 (2002).

[21] M. Parac, S. Grimme, Chem. Phys. 292, 11 (2003).

[22] Gaussian 98, Revision A. 9, M.J. Frisch, G.W. Trucks, H.B. Schlegel, G.E. Scuseria, M.A. Robb, J.R. Cheeseman, V.G. Zakrzewski, J.A. Montgomery, Jr., R.E. Stratmann, J.C. Burant, S. Dapprich, J.M. Millam, A.D. Daniels, K.N. Kudin, M.C. Strain, O. Farkas, J. Tomasi, V. Barone, M. Cossi, R. Cammi, B. Mennucci, C. Pomelli, C. Adamo, S. Clifford, J. Ochterski, G.A. Petersson, P.Y. Ayala, Q. Cui, K. Morokuma, D.K. Malick, A.D. Rabuck, K. Raghavachari, J.B. Foresman, J. Cioslowski, J.V. Ortiz, A.G. Baboul, B.B. Stefanov, G. Liu, A. Liashenko, P. Piskorz, I. Komaromi, R. Gomperts, R.L. Martin, D.J. Fox, T. Keith, M.A. Al-Laham, C.Y. Peng, A. Nanayakkara, M. Challacombe, P.M.W. Gill, B. Johnson, W. Chen, M.W. Wong, J.L. Andres, C. Gonzalez, M. Head-Gordon, E.S. Replogle, J.A. Pople, Gaussian, Inc., Pittsburgh (PA) 1998.

[23] N. Thantu, P.M. Weber, Chem. Phys. Lett. 214, 276 (1993).

[24] E.C.M. Chen, W.E. Wentworth, Mol. Cryst. Liq. Cryst. 171, 271 (1989).

[25] F. Negri, M. Zgierski, J. Chem. Phys. 104, 3486 (1996).

[26] http://srdata.nist.gov/cccbdb/Default.htm. 